\title{
A generalized weak contraction principle with applications to coupled coincidence point problems
}

\author{
Binayak S Choudhury ${ }^{1}$, Nikhilesh Metiya ${ }^{2}$ and Mihai Postolache ${ }^{3^{*}}$
}

\author{
*Correspondence: \\ mihai@mathem.pub.ro \\ ${ }^{3}$ Faculty of Applied Sciences, \\ University Politehnica of Bucharest, \\ 313 Splaiul Independentei, \\ Bucharest, 060042, Romania \\ Full list of author information is \\ available at the end of the article
}

\begin{abstract}
In this paper we establish some coincidence point results for generalized weak contractions with discontinuous control functions. The theorems are proved in metric spaces with a partial order. Our theorems extend several existing results in the current literature. We also discuss several corollaries and give illustrative examples. We apply our result to obtain some coupled coincidence point results which effectively generalize a number of established results.
\end{abstract}

MSC: $54 \mathrm{H} 10 ; 54 \mathrm{H} 25$

Keywords: partially ordered set; control function; coincidence point; coupled coincidence point

\section{Introduction}

In this paper we prove certain coincidence point results in partially ordered metric spaces for functions which satisfy a certain inequality involving three control functions. Two of the control functions are discontinuous. Fixed point theory in partially ordered metric spaces is of relatively recent origin. An early result in this direction is due to Turinici [1], in which fixed point problems were studied in partially ordered uniform spaces. Later, this branch of fixed point theory has developed through a number of works, some of which are in $[2-6]$.

Weak contraction was studied in partially ordered metric spaces by Harjani et al. [3]. In a recent result by Choudhury et al. [2], a generalization of the above result to a coincidence point theorem has been done using three control functions. Here we prove coincidence point results by assuming a weak contraction inequality with three control functions, two of which are not continuous. The results are obtained under two sets of additional conditions. A fixed point theorem is also established. There are several corollaries and two examples. One of the examples shows that the corollaries are properly contained in their respective theorem. The corollaries are generalizations of several existing works.

We apply our result to obtain some coupled coincidence point results. Coupled fixed theorems and coupled coincidence point theorems have appeared prominently in recent literature. Although the concept of coupled fixed points was introduced by Guo et al. [7], starting with the work of Gnana Bhaskar and Lakshmikantham [8], where they established a coupled contraction principle, this line of research has developed rapidly in partially ordered metric spaces. References [9-20] are some examples of these works. There is a 
viewpoint from which coupled fixed and coincidence point theorems can be considered as problems in product spaces [21]. We adopt this approach here. Specifically, we apply our theorem to a product of two metric spaces on which a metric is defined from the metric of the original spaces. We establish a generalization of several results. We also discuss an example which shows that our result is an actual improvement over the results it generalizes.

\section{Mathematical preliminaries}

Let $(X, \preceq)$ be a partially ordered set and $T: X \longrightarrow X$. The mapping $T$ is said to be nondecreasing if for all $x_{1}, x_{2} \in X, x_{1} \preceq x_{2}$ implies $T x_{1} \preceq T x_{2}$ and nonincreasing if for all $x_{1}, x_{2} \in X$, $x_{1} \preceq x_{2}$ implies $T x_{1} \succeq T x_{2}$.

Definition 2.1 ([22]) Let $(X, \preceq)$ be a partially ordered set and $T: X \rightarrow X$ and $G: X \longrightarrow X$. The mapping $T$ is said to be G-nondecreasing if for all $x, y \in X, G x \preceq G y$ implies $T x \preceq T y$ and G-nonincreasing if for all $x, y \in X, G x \preceq G y$ implies $T x \succeq T y$.

Definition 2.2 Two self-mappings $G$ and $T$ of a nonempty set $X$ are said to be commutative if $G T x=T G x$ for all $x \in X$.

Definition 2.3 ([23]) Two self-mappings $G$ and $T$ of a metric space $(X, d)$ are said to be compatible if the following relation holds:

$$
\lim _{n \rightarrow \infty} d\left(G T x_{n}, T G x_{n}\right)=0,
$$

whenever $\left\{x_{n}\right\}$ is a sequence in $X$ such that $\lim _{n \rightarrow \infty} G x_{n}=\lim _{n \rightarrow \infty} T x_{n}=x$ for some $x \in X$ is satisfied.

Definition 2.4 ([24]) Two self-mappings $G$ and $T$ of a nonempty set $X$ are said to be weakly compatible if they commute at their coincidence points; that is, if $G x=T x$ for some $x \in X$, then $G T x=T G x$.

Definition 2.5 ([8]) Let $(X, \preceq)$ be a partially ordered set and $F: X \times X \longrightarrow X$. The mapping $F$ is said to have the mixed monotone property if $F$ is monotone nondecreasing in its first argument and is monotone nonincreasing in its second argument; that is, if

$$
x_{1}, x_{2} \in X, \quad x_{1} \preceq x_{2} \quad \Longrightarrow \quad F\left(x_{1}, y\right) \preceq F\left(x_{2}, y\right) \quad \text { for all } y \in X \text {; }
$$

and

$$
y_{1}, y_{2} \in X, \quad y_{1} \preceq y_{2} \quad \Longrightarrow \quad F\left(x, y_{1}\right) \succeq F\left(x, y_{2}\right) \quad \text { for all } x \in X .
$$

Definition 2.6 ([17]) Let $(X, \preceq)$ be a partially ordered set, $F: X \times X \rightarrow X$ and $g: X \longrightarrow X$. We say that $F$ has the mixed $g$-monotone property if

$$
x_{1}, x_{2} \in X, \quad g x_{1} \preceq g x_{2} \quad \Longrightarrow \quad F\left(x_{1}, y\right) \preceq F\left(x_{2}, y\right) \quad \text { for all } y \in X \text {; }
$$

and

$$
y_{1}, y_{2} \in X, \quad g y_{1} \preceq g y_{2} \quad \Longrightarrow \quad F\left(x, y_{1}\right) \succeq F\left(x, y_{2}\right) \quad \text { for all } x \in X .
$$


Definition 2.7 ([8]) An element $(x, y) \in X \times X$ is called a coupled fixed point of the mapping $F: X \times X \longrightarrow X$ if $F(x, y)=x$ and $F(y, x)=y$.

Definition 2.8 ([17]) An element $(x, y) \in X \times X$ is called a coupled coincidence point of the mappings $F: X \times X \longrightarrow X$ and $g: X \longrightarrow X$ if $F(x, y)=g x$ and $F(y, x)=g y$.

Definition 2.9 ([17]) Let $X$ be a nonempty set. The mappings $g$ and $F$, where $g: X \rightarrow X$ and $F: X \times X \longrightarrow X$, are said to be commutative if $g F(x, y)=F(g x, g y)$ for all $x, y \in X$.

Definition 2.10 ([12]) Let $(X, d)$ be a metric space. The mappings $g$ and $F$, where $g: X \longrightarrow$ $X$ and $F: X \times X \longrightarrow X$, are said to be compatible if the following relations hold:

$$
\lim _{n \rightarrow \infty} d\left(g F\left(x_{n}, y_{n}\right), F\left(g x_{n}, g y_{n}\right)\right)=0, \quad \lim _{n \rightarrow \infty} d\left(g F\left(y_{n}, x_{n}\right), F\left(g y_{n}, g x_{n}\right)\right)=0,
$$

whenever $\left\{x_{n}\right\}$ and $\left\{y_{n}\right\}$ are sequences in $X$ such that $\lim _{n \rightarrow \infty} g x_{n}=\lim _{n \rightarrow \infty} F\left(x_{n}, y_{n}\right)=x$ and $\lim _{n \rightarrow \infty} g y_{n}=\lim _{n \rightarrow \infty} F\left(y_{n}, x_{n}\right)=y$ for some $x, y \in X$ are satisfied.

Definition 2.11 Let $X$ be a nonempty set. The mappings $g$ and $F$, where $g: X \rightarrow X$ and $F: X \times X \longrightarrow X$, are said to be weakly compatible if they commute at their coupled coincidence points, that is, if $F(x, y)=g x$ and $F(y, x)=g y$ for some $(x, y) \in X \times X$, then $g F(x, y)=F(g x, g y)$ and $g F(y, x)=F(g y, g x)$.

Definition 2.12 ([25]) A function $\psi:[0, \infty) \rightarrow[0, \infty)$ is called an altering distance function if the following properties are satisfied:

(i) $\psi$ is monotone increasing and continuous;

(ii) $\psi(t)=0$ if and only if $t=0$.

In our results in the following sections, we use the following classes of functions.

We denote by $\Psi$ the set of all functions $\psi:[0, \infty) \longrightarrow[0, \infty)$ satisfying

$\left(\mathrm{i}_{\psi}\right) \psi$ is continuous and monotone non-decreasing,

(ii $\psi) \psi(t)=0$ if and only if $t=0$;

and by $\Theta$ we denote the set of all functions $\alpha:[0, \infty) \longrightarrow[0, \infty)$ such that

$\left(\mathrm{i}_{\alpha}\right) \quad \alpha$ is bounded on any bounded interval in $[0, \infty)$,

(ii $\alpha) \alpha$ is continuous at 0 and $\alpha(0)=0$.

\section{Main results}

Let $(X, \preceq, d)$ be an ordered metric space. $X$ is called regular if it has the following properties:

(i) if a nondecreasing sequence $\left\{x_{n}\right\} \longrightarrow x$ in $(X, d)$, then $x_{n} \preceq x$ for all $n \geq 0$;

(ii) if a nonincreasing sequence $\left\{y_{n}\right\} \longrightarrow y$ in $(X, d)$, then $y \preceq y_{n}$ for all $n \geq 0$.

Theorem 3.1 Let $(X, \preceq)$ be a partially ordered set and suppose that there is a metric $d$ on $X$ such that $(X, d)$ is a complete metric space. Let $T, G: X \longrightarrow X$ be two mappings such that $G$ is continuous and nondecreasing, $T(X) \subseteq G(X), T$ is G-nondecreasing with respect to $\preceq$ and the pair $(G, T)$ is compatible. Suppose that there exist $\psi \in \Psi$ and $\varphi, \theta \in \Theta$ such that

$$
\psi(x) \leq \varphi(y) \quad \Longrightarrow \quad x \leq y
$$


for any sequence $\left\{x_{n}\right\}$ in $[0, \infty)$ with $x_{n} \longrightarrow t>0$,

$$
\psi(t)-\varlimsup \varphi\left(x_{n}\right)+\underline{\lim } \theta\left(x_{n}\right)>0,
$$

and for all $x, y \in X$ with $G x \succeq G y$

$$
\psi(d(T x, T y)) \leq \varphi(d(G x, G y))-\theta(d(G x, G y)) .
$$

\section{Also, suppose that}

(a) $T$ is continuous, or

(b) $X$ is regular.

If there exists $x_{0} \in X$ such that $G x_{0} \preceq T x_{0}$, then $G$ and $T$ have a coincidence point in $X$.

Proof Let $x_{0} \in X$ be such that $G x_{0} \preceq T x_{0}$. Since $T(X) \subseteq G(X)$, we can choose $x_{1} \in X$ such that $G x_{1}=T x_{0}$. Again, we can choose $x_{2} \in X$ such that $G x_{2}=T x_{1}$. Continuing this process, we construct a sequence $\left\{x_{n}\right\}$ in $X$ such that

$$
G x_{n+1}=T x_{n} \quad \text { for all } n \geq 0 .
$$

Since $G x_{0} \preceq T x_{0}$ and $G x_{1}=T x_{0}$, we have $G x_{0} \preceq G x_{1}$, which implies that $T x_{0} \preceq T x_{1}$. Now, $T x_{0} \preceq T x_{1}$, that is, $G x_{1} \preceq G x_{2}$ implies that $T x_{1} \preceq T x_{2}$. Again, $T x_{1} \preceq T x_{2}$, that is, $G x_{2} \preceq G x_{3}$ implies that $T x_{2} \preceq T x_{3}$. Continuing this process, we have

$$
G x_{0} \preceq G x_{1} \preceq G x_{2} \preceq G x_{3} \preceq \cdots \preceq G x_{n} \preceq G x_{n+1} \preceq \cdots,
$$

and

$$
T x_{0} \preceq T x_{1} \preceq T x_{2} \preceq T x_{3} \preceq \cdots \preceq T x_{n} \preceq T x_{n+1} \preceq \cdots
$$

Let $R_{n}=d\left(G x_{n+1}, G x_{n}\right)$ for all $n \geq 0$.

Since $G x_{n+1} \succeq G x_{n}$, from (3.3) and (3.4), we have

$$
\psi\left(d\left(G x_{n+2}, G x_{n+1}\right)\right)=\psi\left(d\left(T x_{n+1}, T x_{n}\right)\right) \leq \varphi\left(d\left(G x_{n+1}, G x_{n}\right)\right)-\theta\left(d\left(G x_{n+1}, G x_{n}\right)\right),
$$

that is,

$$
\psi\left(R_{n+1}\right) \leq \varphi\left(R_{n}\right)-\theta\left(R_{n}\right),
$$

which, in view of the fact that $\theta \geq 0$, yields $\psi\left(R_{n+1}\right) \leq \varphi\left(R_{n}\right)$, which by (3.1) implies that $R_{n+1} \leq R_{n}$ for all positive integer $n$, that is, $\left\{R_{n}\right\}$ is a monotone decreasing sequence. Hence there exists an $r \geq 0$ such that

$$
R_{n}=d\left(G x_{n+1}, G x_{n}\right) \longrightarrow r \quad \text { as } n \longrightarrow \infty .
$$

Taking limit supremum on both sides of (3.7), using (3.8), the property ( $\left.\mathrm{i}_{\alpha}\right)$ of $\varphi$ and $\theta$, and the continuity of $\psi$, we obtain

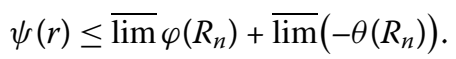


Since $\overline{\lim }\left(-\theta\left(R_{n}\right)\right)=-\underline{\lim } \theta\left(R_{n}\right)$, it follows that

$$
\psi(r) \leq \varlimsup-\underline{\lim } \varphi\left(R_{n}\right)-\underline{\lim } \theta\left(R_{n}\right),
$$

that is,

$$
\psi(r)-\varlimsup \varphi\left(R_{n}\right)+\underline{\lim } \theta\left(R_{n}\right) \leq 0,
$$

which by (3.2) is a contradiction unless $r=0$. Therefore,

$$
R_{n}=d\left(G x_{n+1}, G x_{n}\right) \longrightarrow 0 \quad \text { as } n \longrightarrow \infty .
$$

Next we show that $\left\{G x_{n}\right\}$ is a Cauchy sequence.

Suppose that $\left\{G x_{n}\right\}$ is not a Cauchy sequence. Then there exists an $\epsilon>0$ for which we can find two sequences of positive integers $\{m(k)\}$ and $\{n(k)\}$ such that for all positive integers $k, n(k)>m(k)>k$ and $d\left(G x_{m(k)}, G x_{n(k)}\right) \geq \epsilon$. Assuming that $n(k)$ is the smallest such positive integer, we get

$$
\begin{aligned}
& n(k)>m(k)>k, \\
& d\left(G x_{m(k)}, G x_{n(k)}\right) \geq \epsilon \text { and } \\
& d\left(G x_{m(k)}, G x_{n(k)-1}\right)<\epsilon .
\end{aligned}
$$

Now,

$$
\epsilon \leq d\left(G x_{m(k)}, G x_{n(k)}\right) \leq d\left(G x_{m(k)}, G x_{n(k)-1}\right)+d\left(G x_{n(k)-1}, G x_{n(k)}\right),
$$

that is,

$$
\epsilon \leq d\left(G x_{m(k)}, G x_{n(k)}\right) \leq \epsilon+d\left(G x_{n(k)-1}, G x_{n(k)}\right) .
$$

Letting $k \longrightarrow \infty$ in the above inequality and using (3.9), we have

$$
\lim _{k \rightarrow \infty} d\left(G x_{m(k)}, G x_{n(k)}\right)=\epsilon
$$

Again,

$$
d\left(G x_{m(k)+1}, G x_{n(k)+1}\right) \leq d\left(G x_{m(k)+1}, G x_{m(k)}\right)+d\left(G x_{m(k)}, G x_{n(k)}\right)+d\left(G x_{n(k)+1}, G x_{n(k)}\right)
$$

and

$$
d\left(G x_{m(k)}, G x_{n(k)}\right) \leq d\left(G x_{m(k)+1}, G x_{m(k)}\right)+d\left(G x_{m(k)+1}, G x_{n(k)+1}\right)+d\left(G x_{n(k)+1}, G x_{n(k)}\right) .
$$

Letting $k \longrightarrow \infty$ in the above inequalities, using (3.9) and (3.10), we have

$$
\lim _{k \rightarrow \infty} d\left(G x_{m(k)+1}, G x_{n(k)+1}\right)=\epsilon .
$$


As $n(k)>m(k), G x_{n(k)} \succeq G x_{m(k)}$, from (3.3) and (3.4), we have

$$
\begin{aligned}
\psi\left(d\left(G x_{n(k)+1}, G x_{m(k)+1}\right)\right) & =\psi\left(d\left(T x_{n(k)}, T x_{m(k)}\right)\right) \\
& \leq \varphi\left(d\left(G x_{n(k)}, G x_{m(k)}\right)\right)-\theta\left(d\left(G x_{n(k)}, G x_{m(k)}\right)\right)
\end{aligned}
$$

Taking limit supremum on both sides of the above inequality, using (3.10), (3.11), the property $\left(\mathrm{i}_{\alpha}\right)$ of $\varphi$ and $\theta$, and the continuity of $\psi$, we obtain

$$
\psi(\epsilon) \leq \varlimsup \lim \varphi\left(d\left(G x_{n(k)}, G x_{m(k)}\right)\right)+\varlimsup\left(-\theta\left(d\left(G x_{n(k)}, G x_{m(k)}\right)\right)\right) .
$$

Since $\varlimsup\left(-\theta\left(d\left(G x_{n(k)}, G x_{m(k)}\right)\right)\right)=-\underline{\lim } \theta\left(d\left(G x_{n(k)}, G x_{m(k)}\right)\right)$, it follows that

$$
\psi(\epsilon) \leq \varlimsup \lim \varphi\left(d\left(G x_{n(k)}, G x_{m(k)}\right)\right)-\underline{\lim } \theta\left(d\left(G x_{n(k)}, G x_{m(k)}\right)\right),
$$

that is,

$$
\psi(\epsilon)-\varlimsup \varphi\left(d\left(G x_{n(k)}, G x_{m(k)}\right)\right)+\underline{\lim } \theta\left(d\left(G x_{n(k)}, G x_{m(k)}\right)\right) \leq 0,
$$

which is a contradiction by (3.2). Therefore, $\left\{G x_{n}\right\}$ is a Cauchy sequence in $X$. From the completeness of $X$, there exists $x \in X$ such that

$$
\lim _{n \rightarrow \infty} T x_{n}=\lim _{n \rightarrow \infty} G x_{n}=x
$$

Since the pair $(G, T)$ is compatible, from (3.12), we have

$$
\lim _{n \rightarrow \infty} d\left(G T x_{n}, T G x_{n}\right)=0
$$

Let the condition (a) hold.

By the triangular inequality, we have

$$
d\left(G x, T G x_{n}\right) \leq d\left(G x, G T x_{n}\right)+d\left(G T x_{n}, T G x_{n}\right) .
$$

Taking $n \longrightarrow \infty$ in the above inequality, using (3.12), (3.13) and the continuities of $T$ and $G$, we have $d(G x, T x)=0$, that is, $G x=T x$, that is, $x$ is a coincidence point of the mappings $G$ and $T$.

Next we suppose that the condition (b) holds.

By (3.5) and (3.12), we have $G x_{n} \preceq x$ for all $n \geq 0$. Using the monotone property of $G$, we obtain

$$
G G x_{n} \preceq G x .
$$

As $G$ is continuous and the pair $(G, T)$ is compatible, by (3.12) and (3.13), we have

$$
\lim _{n \rightarrow \infty} G G x_{n}=G x=\lim _{n \rightarrow \infty} G T x_{n}=\lim _{n \rightarrow \infty} T G x_{n} .
$$


Then

$$
d(T x, G x)=\lim _{n \rightarrow \infty} d\left(T x, T G x_{n}\right) .
$$

Since $\psi$ is continuous, from the above inequality, we obtain

$$
\psi(d(T x, G x))=\psi\left(\lim _{n \rightarrow \infty} d\left(T x, T G x_{n}\right)\right)=\lim _{n \rightarrow \infty} \psi\left(d\left(T x, T G x_{n}\right)\right),
$$

which, by (3.3) and (3.14), implies that

$$
\psi(d(T x, G x)) \leq \lim _{n \rightarrow \infty}\left[\varphi\left(d\left(G x, G G x_{n}\right)\right)-\theta\left(d\left(G x, G G x_{n}\right)\right)\right] .
$$

Using (3.15) and the property ( $\mathrm{ii}_{\alpha}$ ) of $\varphi$ and $\theta$, we have

$$
\psi(d(T x, G x))=0
$$

which, by the property of $\psi$, implies that $d(T x, G x)=0$, that is, $G x=T x$, that is, $x$ is a coincidence point of the mappings $G$ and $T$.

Next we discuss some corollaries of Theorem 3.1. By an example, we show that Theorem 3.1 properly contains all its corollaries.

Every commuting pair $(G, T)$ is also a compatible pair. Then considering $(G, T)$ to be the commuting pair in Theorem 3.1, we have the following corollary.

Corollary 3.1 Let $(X, \preceq)$ be a partially ordered set and suppose that there is a metric $d$ on $X$ such that $(X, d)$ is a complete metric space. Let $T, G: X \longrightarrow X$ be two mappings such that $G$ is continuous and nondecreasing, $T(X) \subseteq G(X), T$ is G-nondecreasing with respect to $\preceq$ and the pair $(G, T)$ is commutative. Suppose that there exist $\psi \in \Psi$ and $\varphi, \theta \in \Theta$ such that (3.1), (3.2) and (3.3) are satisfied. Also, suppose that

(a) $T$ is continuous, or

(b) $X$ is regular.

If there exists $x_{0} \in X$ such that $G x_{0} \preceq T x_{0}$, then $G$ and $T$ have a coincidence point in $X$.

Considering $\psi$ to be the identity mapping and $\theta(t)=0$ for all $t \in[0, \infty)$ in Theorem 3.1, we have the following corollary.

Corollary 3.2 Let $(X, \preceq)$ be a partially ordered set and suppose that there is a metric $d$ on $X$ such that $(X, d)$ is a complete metric space. Let $T, G: X \longrightarrow X$ be two mappings such that $G$ is continuous and nondecreasing, $T(X) \subseteq G(X), T$ is $G$-nondecreasing with respect to $\preceq$ and the pair $(G, T)$ is compatible. Suppose that there exists $\varphi \in \Theta$ such that for any sequence $\left\{x_{n}\right\}$ in $[0, \infty)$ with $x_{n} \longrightarrow t>0$,

$$
\varlimsup \varphi\left(x_{n}\right)<t
$$

and for all $x, y \in X$ with $G x \geq G y$,

$$
d(T x, T y) \leq \varphi(d(G x, G y)) .
$$


Also, suppose that

(a) $T$ is continuous, or

(b) $X$ is regular.

If there exists $x_{0} \in X$ such that $G x_{0} \preceq T x_{0}$, then $G$ and $T$ have a coincidence point in $X$.

Considering $\theta(t)=0$ for all $t \in[0, \infty)$ and $\varphi(t)=k \psi(t)$ with $k \in[0,1)$ in Theorem 3.1, we have the following corollary.

Corollary 3.3 Let $(X, \preceq)$ be a partially ordered set and suppose that there is a metric $d$ on $X$ such that $(X, d)$ is a complete metric space. Let $T, G: X \rightarrow X$ be two mappings such that $G$ is continuous and nondecreasing, $T(X) \subseteq G(X), T$ is G-nondecreasing with respect to $\preceq$ and the pair $(G, T)$ is compatible. Suppose that there exists $\psi \in \Psi$ and $k \in[0,1)$ such that for all $x, y \in X$ with $G x \geq G y$,

$$
\psi(d(T x, T y)) \leq k \psi(d(G x, G y)) .
$$

Also, suppose that

(a) $T$ is continuous, or

(b) $X$ is regular.

If there exists $x_{0} \in X$ such that $G x_{0} \preceq T x_{0}$, then $G$ and $T$ have a coincidence point in $X$.

Considering $\varphi$ to be the function $\psi$ in Theorem 3.1, we have the following corollary.

Corollary 3.4 Let $(X, \preceq)$ be a partially ordered set and suppose that there is a metric $d$ on $X$ such that $(X, d)$ is a complete metric space. Let $T, G: X \rightarrow X$ be two mappings such that $G$ is continuous and nondecreasing, $T(X) \subseteq G(X), T$ is G-nondecreasing with respect to $\preceq$ and the pair $(G, T)$ is compatible. Suppose that there exist $\psi \in \Psi$ and $\theta \in \Theta$ such that for any sequence $\left\{x_{n}\right\}$ in $[0, \infty)$ with $x_{n} \longrightarrow t>0$,

$$
\underline{\lim } \theta\left(x_{n}\right)>0
$$

and for all $x, y \in X$ with $G x \succeq G y$,

$$
\psi(d(T x, T y)) \leq \psi(d(G x, G y))-\theta(d(G x, G y))
$$

Also, suppose that

(a) $T$ is continuous or

(b) $X$ is regular.

If there exists $x_{0} \in X$ such that $G x_{0} \preceq T x_{0}$, then $G$ and $T$ have a coincidence point in $X$.

If $\psi$ and $\varphi$ are the identity mappings in Theorem 3.1, we have the following corollary.

Corollary 3.5 Let $(X, \preceq)$ be a partially ordered set and suppose that there is a metric $d$ on $X$ such that $(X, d)$ is a complete metric space. Let $T, G: X \longrightarrow X$ be two mappings such that $G$ is continuous and nondecreasing, $T(X) \subseteq G(X), T$ is G-nondecreasing with respect 
to $\preceq$ and the pair $(G, T)$ is compatible. Suppose that there exists $\theta \in \Theta$ such that for any sequence $\left\{x_{n}\right\}$ in $[0, \infty)$ with $x_{n} \longrightarrow t>0, \underline{\lim } \theta\left(x_{n}\right)>0$ and for all $x, y \in X$ with $G x \geq G y$,

$$
d(T x, T y) \leq d(G x, G y)-\theta(d(G x, G y)) .
$$

Also, suppose that

(a) $T$ is continuous, or

(b) $X$ is regular.

If there exists $x_{0} \in X$ such that $G x_{0} \leq T x_{0}$, then $G$ and $T$ have a coincidence point in $X$.

Considering $\psi$ and $\varphi$ to be the identity mappings and $\theta(t)=(1-k) t$, where $0 \leq k<1$ in Theorem 3.1, we have the following corollary.

Corollary 3.6 Let $(X, \preceq)$ be a partially ordered set and suppose that there is a metric $d$ on $X$ such that $(X, d)$ is a complete metric space. Let $T, G: X \rightarrow X$ be two mappings such that $G$ is continuous and nondecreasing, $T(X) \subseteq G(X), T$ is $G$-nondecreasing with respect to $\preceq$ and the pair $(G, T)$ is compatible. Assume that there exists $k \in[0,1)$ such that for all $x, y \in X$ with $G x \succeq G y$,

$$
d(T x, T y) \leq k d(G x, G y) .
$$

Also, suppose that

(a) $T$ is continuous, or

(b) $X$ is regular.

If there exists $x_{0} \in X$ such that $G x_{0} \preceq T x_{0}$, then $G$ and $T$ have a coincidence point in $X$.

The condition (i), the continuity and the monotone property of the function $G$, and (ii), the compatibility condition of the pairs $(G, T)$, which were considered in Theorem 3.1, are relaxed in our next theorem by taking $G(X)$ to be closed in $(X, d)$.

Theorem 3.2 Let $(X, \preceq)$ be a partially ordered set and suppose that there is a metric $d$ on $X$ such that $(X, d)$ is a complete metric space. Let $T, G: X \longrightarrow X$ be two mappings such that $T(X) \subseteq G(X)$ and $T$ is G-nondecreasing with respect to $\preceq$ and $G(X)$ is closed in $X$. Suppose that there exist $\psi \in \Psi$ and $\varphi, \theta \in \Theta$ such that (3.1), (3.2) and (3.3) are satisfied. Also, suppose that $X$ is regular.

If there exists $x_{0} \in X$ such that $G x_{0} \preceq T x_{0}$, then $G$ and $T$ have a coincidence point in $X$.

Proof We take the same sequence $\left\{x_{n}\right\}$ as in the proof of Theorem 3.1. Then we have (3.12), that is,

$$
\lim _{n \rightarrow \infty} T x_{n}=\lim _{n \rightarrow \infty} G x_{n}=x
$$

Since $\left\{G x_{n}\right\}$ is a sequence in $G(X)$ and $G(X)$ is closed in $X, x \in G(X)$. As $x \in G(X)$, there exists $z \in X$ such that $x=G z$. Then

$$
\lim _{n \rightarrow \infty} T x_{n}=\lim _{n \rightarrow \infty} G x_{n}=G z .
$$


Now, $\left\{G x_{n}\right\}$ is nondecreasing and converges to $G z$. So, by the order condition of the metric space $X$, we have

$$
G x_{n} \preceq G z .
$$

Putting $x=z$ and $y=x_{n}$ in (3.3), by the virtue of (3.24), we get

$$
\psi\left(d\left(T z, T x_{n}\right)\right) \leq \varphi\left(d\left(G z, G x_{n}\right)\right)-\theta\left(d\left(G z, G x_{n}\right)\right) .
$$

Taking $n \rightarrow \infty$ in the above inequality, using (3.23), the property $\left(\mathrm{ii}_{\alpha}\right)$ of $\varphi$ and $\theta$ and the continuity of $\psi$, we have

$$
\psi(d(T z, G z))=0
$$

which, by the property of $\psi$, implies that $d(T z, G z)=0$, that is, $T z=G z$, that is, $z$ is a coincidence point of the mappings $G$ and $T$.

In the following, our aim is to prove the existence and uniqueness of the common fixed point in Theorems 3.1 and 3.2.

Theorem 3.3 In addition to the hypotheses of Theorems 3.1 and 3.2, in both of the theorems, suppose that for every $x, y \in X$ there exists $u \in X$ such that Tu is comparable to $T x$ and $T y$, and also the pair $(G, T)$ is weakly compatible. Then $G$ and $T$ have a unique common fixed point.

Proof From Theorem 3.1 or Theorem 3.2, the set of coincidence points of $G$ and $T$ is nonempty. Suppose $x$ and $y$ are coincidence points of $G$ and $T$, that is, $G x=T x$ and $G y=T y$. Now, we show

$$
G x=G y .
$$

By the assumption, there exists $u \in X$ such that $T u$ is comparable with $T x$ and $T y$.

Put $u_{0}=u$ and choose $u_{1} \in X$ so that $G u_{1}=T u_{0}$. Then, similarly to the proof of Theorem 3.1, we can inductively define sequences $\left\{G u_{n}\right\}$ where $G u_{n+1}=T u_{n}$ for all $n \geq 0$. Hence $T x=G x$ and $T u=T u_{0}=G u_{1}$ are comparable.

Suppose that $G u_{1} \preceq G x$ (the proof is similar to that in the other case).

We claim that $G u_{n} \preceq G x$ for each $n \in N$.

In fact, we will use mathematical induction. Since $G u_{1} \preceq G x$, our claim is true for $n=1$. We presume that $G u_{n} \preceq G x$ holds for some $n>1$. Since $T$ is $G$-nondecreasing with respect to $\preceq$, we get

$$
G u_{n+1}=T u_{n} \preceq T x=G x
$$

and this proves our claim.

Let $R_{n}=d\left(G x, G u_{n}\right)$. Since $G u_{n} \preceq G x$, using the contractive condition (3.3), for all $n \geq 1$, we have

$$
\psi\left(d\left(G x, G u_{n+1}\right)\right)=\psi\left(d\left(T x, T u_{n}\right)\right) \leq \varphi\left(d\left(G x, G u_{n}\right)\right)-\theta\left(d\left(G x, G u_{n}\right)\right),
$$


that is, $\psi\left(R_{n+1}\right) \leq \varphi\left(R_{n}\right)-\theta\left(R_{n}\right)$, which, in view of the fact that $\theta \geq 0$, yields $\psi\left(R_{n+1}\right) \leq$ $\varphi\left(R_{n}\right)$, which by (3.1) implies that $R_{n+1} \leq R_{n}$ for all positive integer $n$, that is, $\left\{R_{n}\right\}$ is a monotone decreasing sequence.

Then, as in the proof of Theorem 3.1, we have

$$
\lim _{n \rightarrow \infty} R_{n}=\lim _{n \rightarrow \infty} d\left(G x, G u_{n}\right)=0
$$

Similarly, we show that

$$
\lim _{n \rightarrow \infty} d\left(G y, G u_{n}\right)=0 .
$$

By the triangle inequality, using (3.26) and (3.27), we have

$$
d(G x, G y) \leq\left[d\left(G x, G u_{n}\right)+d\left(G u_{n}, G y\right)\right] \longrightarrow 0 \quad \text { as } n \longrightarrow \infty .
$$

Hence $G x=G y$. Thus (3.25) is proved.

Since $G x=T x$, by weak compatibility of $G$ and $T$, we have

$$
G G x=G T x=T G x .
$$

Denote

$$
G x=z
$$

Then from (3.28) we have

$$
G z=T z .
$$

Thus $z$ is a coincidence point of $G$ and $T$. Then from (3.25) with $y=z$ it follows that

$$
G x=G z .
$$

By (3.29) it follows that

$$
z=G z
$$

From (3.29) and (3.30), we get $z=G z=T z$.

Therefore, $z$ is a common fixed point of $G$ and $T$.

To prove the uniqueness, assume that $r$ is another common fixed point of $G$ and $T$. Then by (3.25) we have $r=G r=G z=z$. Hence the common fixed point of $G$ and $T$ is unique.

Example 3.1 Let $X=[0, \infty)$. Then $(X, \leq)$ is a partially ordered set with the natural ordering of real numbers. Let $d(x, y)=|x-y|$ for $x, y \in X$. Then $(X, d)$ is a complete metric space.

Let $T, G: X \rightarrow X$ be given respectively by the formulas $T x=\frac{1}{3} x^{2}$ and $G x=x^{2}$ for all $x \in X$. Then $T$ and $G$ satisfy all the properties mentioned in Theorem 3.1. 
Let $\psi, \varphi, \theta:[0, \infty) \longrightarrow[0, \infty)$ be given respectively by the formulas

$$
\psi(t)=t^{2}, \quad \varphi(t)=\left\{\begin{array}{ll}
\frac{1}{2}[t]^{2} & \text { if } 3<t<4, \\
\frac{4}{9} t^{2} & \text { otherwise, }
\end{array} \quad \theta(t)= \begin{cases}\frac{1}{9}[t]^{2} & \text { if } 3<t<4 \\
0 & \text { otherwise }\end{cases}\right.
$$

Then $\psi, \varphi$ and $\theta$ have the properties mentioned in Theorem 3.1.

It can be verified that (3.3) is satisfied for all $x, y \in X$ with $G x \succeq G y$. Hence the required conditions of Theorem 3.1 are satisfied and it is seen that 0 is a coincidence point of $G$ and $T$. Also, the conditions of Theorem 3.3 are satisfied and it is seen that 0 is the unique common fixed point of $G$ and $T$.

Remark 3.1 In the above example, the pair $(G, T)$ is compatible but not commuting so that Corollary 3.1 is not applicable to this example and hence Theorem 3.1 properly contains its Corollary 3.1.

Remark 3.2 In the above example, $\psi$ is not the identity mapping and $\theta(t) \neq 0$ for all $t$ in $[0, \infty)$. Let us consider the sequence $\left\{x_{n}\right\}$ in $[0, \infty)$, where $x_{n}=3$ for all $n$. Then $\varphi\left(x_{n}\right)=4$ for all $n$. Now $x_{n} \longrightarrow t=3>0$, but $\varlimsup \varphi\left(x_{n}\right)=4 \nless t=3$. Therefore, Corollary 3.2 is not applicable to this example, and hence Theorem 3.1 properly contains its Corollary 3.2.

Remark 3.3 The above example $\theta(t) \neq 0$ for all $t \in[0, \infty)$, and hence Corollary 3.3 is not applicable to the example, and so Theorem 3.1 properly contains its Corollary 3.3.

Remark 3.4 In the above example, $\varphi$ is not identical to the function $\psi$, and also $\underline{\lim } \theta\left(x_{n}\right) \ngtr 0$ for any sequence $\left\{x_{n}\right\}$ in $[0, \infty)$ with $x_{n} \longrightarrow t>0$. Therefore, Corollaries 3.4 and 3.5 are not applicable to this example, and hence Theorem 3.1 properly contains its Corollaries 3.4 and 3.5.

Remark 3.5 In the above example, $\psi$ and $\varphi$ are not the identity functions and $\theta(t) \neq(1-$ $k) t$ with $0 \leq k<1$. Therefore, Corollary 3.6 is not applicable to the above example. Hence Theorem 3.1 properly contains its Corollary 3.6.

Remark 3.6 Theorem 3.1 generalizes the results in [2-4, 6, 25-29].

Example 3.2 Let $X=[0,1]$. Then $(X, \leq)$ is a partially ordered set with the natural ordering of real numbers. Let $d(x, y)=|x-y|$ for $x, y \in X$. Then $(X, d)$ is a metric space with the required properties of Theorem 3.2.

Let $T, G: X \rightarrow X$ be given respectively by the formulas

$$
T x=1 \text { for all } x \in X, \quad G x= \begin{cases}1 & \text { if } x \text { is rational, } \\ \frac{1}{2} & \text { if } x \text { is irrational. }\end{cases}
$$

Then $T$ and $G$ have the properties mentioned in Theorem 3.2.

Let $\psi, \varphi, \theta:[0, \infty) \longrightarrow[0, \infty)$ be given respectively by the formulas

$$
\psi(t)=t^{2}, \quad \varphi(t)=\left\{\begin{array}{ll}
\frac{1}{2}[t]^{2} & \text { if } 3<t<4, \\
\frac{4}{9} t^{2} & \text { otherwise, }
\end{array} \quad \theta(t)= \begin{cases}\frac{1}{9}[t]^{2} & \text { if } 3<t<4, \\
0 & \text { otherwise }\end{cases}\right.
$$

Then $\psi, \varphi$ and $\theta$ have the properties mentioned in Theorem 3.2. 
All the required conditions of Theorem 3.2 are satisfied. It is seen that every rational number $x \in X$ is a coincidence point of $G$ and $T$. Also, the conditions of Theorem 3.3 are satisfied and it is seen that 1 is the unique common fixed point of $G$ and $T$.

Remark 3.7 In the above example, the function $g$ is not continuous. Therefore, Theorem 3.1 is not applicable to the above example.

\section{Applications to coupled coincidence point results}

In this section, we use the results of the previous section to establish new coupled coincidence point results in partially ordered metric spaces. Our results are extensions of some existing results.

Let $(X, \preceq)$ be a partially ordered set. Now, we endow the product space $X \times X$ with the following partial order:

$$
\text { for }(x, y),(u, v) \in X \times X, \quad(u, v) \preceq(x, y) \quad \Leftrightarrow \quad x \succeq u, y \preceq v .
$$

Let $(X, d)$ be a metric space. Then $d_{1}$ given by the law

$$
d_{1}((x, y),(u, v))=d(x, u)+d(y, v) \quad \text { for }(x, y),(u, v) \in X \times X
$$

is a metric on $X \times X$.

Let $g: X \longrightarrow X$ and $F: X \times X \rightarrow X$ be two mappings. Then we define two functions $G: X \times X \longrightarrow X \times X$ and $T: X \times X \longrightarrow X \times X$ respectively as follows:

$$
G(x, y)=(g x, g y) \quad \text { for } x, y \in X, \quad T(x, y)=(F(x, y), F(y, x)) \quad \text { for } x, y \in X .
$$

Lemma 4.1 Let $(X, \preceq)$ be a partially ordered set, $F: X \times X \rightarrow X$ and $g: X \longrightarrow X$. If $F$ has the mixed $g$-monotone property, then $T$ is G-nondecreasing.

Proof Let $\left(x_{1}, y_{1}\right),\left(x_{2}, y_{2}\right) \in X \times X$ such that $G\left(x_{1}, y_{1}\right) \preceq G\left(x_{2}, y_{2}\right)$. Then, by the definition of $G$, it follows that $\left(g x_{1}, g y_{1}\right) \preceq\left(g x_{2}, g y_{2}\right)$, that is, $g x_{1} \preceq g x_{2}$ and $g y_{1} \succeq g y_{2}$. Since $F$ has the mixed $g$-monotone property, we have

$$
F\left(x_{1}, y_{1}\right) \preceq F\left(x_{2}, y_{1}\right) \preceq F\left(x_{2}, y_{2}\right),
$$

and

$$
F\left(y_{1}, x_{1}\right) \succeq F\left(y_{2}, x_{1}\right) \succeq F\left(y_{2}, x_{2}\right) .
$$

It follows that $\left(F\left(x_{1}, y_{1}\right), F\left(y_{1}, x_{1}\right)\right) \preceq\left(F\left(x_{2}, y_{2}\right), F\left(y_{2}, x_{2}\right)\right)$, that is, $T\left(x_{1}, y_{1}\right) \preceq T\left(x_{2}, y_{2}\right)$. Therefore, $T$ is $G$-nondecreasing.

Lemma 4.2 Let $X$ be a nonempty set, $g: X \longrightarrow X$ and $F: X \times X \rightarrow X$. If $g$ and $F$ are commutative, then the mappings $G$ and $T$ are also commutative. 
Proof Let $(x, y) \in X \times X$. Since $g$ and $F$ are commutative, by the definition of $G$ and $T$, we have

$$
\begin{aligned}
G T(x, y) & =G(F(x, y), F(y, x))=(g F(x, y), g F(y, x))=(F(g x, g y), F(g y, g x)) \\
& =T(g x, g y)=T G(x, y),
\end{aligned}
$$

which shows that $G$ and $T$ are commutative.

Lemma 4.3 Let $(X, d)$ be metric space and $g: X \longrightarrow X, F: X \times X \longrightarrow X$. If $g$ and $F$ are compatible, then the mappings $G$ and $T$ are also compatible.

Proof Let $\left\{\left(x_{n}, y_{n}\right)\right\}$ be a sequence in $X \times X$ such that $\lim _{n \rightarrow \infty} G\left(x_{n}, y_{n}\right)=\lim _{n \rightarrow \infty} T\left(x_{n}, y_{n}\right)=$ $(x, y)$ for some $(x, y) \in X \times X$. By the definition of $G$ and $T$, we have $\lim _{n \rightarrow \infty}\left(g x_{n}, g y_{n}\right)=$ $\lim _{n \rightarrow \infty}\left(F\left(x_{n}, y_{n}\right), F\left(y_{n}, y_{n}\right)\right)=(x, y)$, which implies that

$$
\lim _{n \rightarrow \infty} F\left(x_{n}, y_{n}\right)=\lim _{n \rightarrow \infty} g x_{n}=x, \quad \lim _{n \rightarrow \infty} F\left(y_{n}, x_{n}\right)=\lim _{n \rightarrow \infty} g y_{n}=y .
$$

Now

$$
\begin{aligned}
d_{1}\left(G T\left(x_{n}, y_{n}\right), T G\left(x_{n}, y_{n}\right)\right) & =d_{1}\left(G\left(F\left(x_{n}, y_{n}\right), F\left(y_{n}, x_{n}\right)\right), T\left(g x_{n}, g y_{n}\right)\right) \\
& =d_{1}\left(\left(g F\left(x_{n}, y_{n}\right), g F\left(y_{n}, x_{n}\right)\right),\left(F\left(g x_{n}, g y_{n}\right), F\left(g y_{n}, g x_{n}\right)\right)\right) \\
& =d\left(g F\left(x_{n}, y_{n}\right), F\left(g x_{n}, g y_{n}\right)\right)+d\left(g F\left(y_{n}, x_{n}\right), F\left(g y_{n}, g x_{n}\right)\right) .
\end{aligned}
$$

Since $g$ and $F$ are compatible, we have

$$
\lim _{n \rightarrow \infty} d_{1}\left(G T\left(x_{n}, y_{n}\right), T G\left(x_{n}, y_{n}\right)\right)=0
$$

It follows that $G$ and $T$ are compatible.

Lemma 4.4 Let $X$ be a nonempty set, $g: X \longrightarrow X$ and $F: X \times X \longrightarrow X$. If $g$ and $F$ are weak compatible, then the mappings $G$ and $T$ are also weak compatible.

Proof Let $(x, y) \in X \times X$ be a coincidence point $G$ and $T$. Then $G(x, y)=T(x, y)$, that is, $(g x, g y)=(F(x, y), F(y, x))$, that is, $g x=F(x, y)$ and $g y=F(y, x)$. Since $g$ and $F$ are weak compatible, by the definition of $G$ and $T$, we have

$$
\begin{aligned}
G T(x, y) & =G(F(x, y), F(y, x))=(g F(x, y), g F(y, x)) \\
& =(F(g x, g y), F(g y, g x))=T(g x, g y)=T G(x, y),
\end{aligned}
$$

which shows that $G$ and $T$ commute at their coincidence point, that is, $G$ and $T$ are weak compatible.

Theorem 4.1 Let $(X, \preceq)$ be a partially ordered set and suppose that there is a metric $d$ on $X$ such that $(X, d)$ is a complete metric space. Let $F: X \times X \longrightarrow X$ and $g: X \rightarrow X$ be two mappings such that $g$ is continuous and nondecreasing, $F(X \times X) \subseteq g(X)$, F has the mixed $g$-monotone property on $X$ and the pair $(g, F)$ is compatible. Suppose that there exist $\psi \in \Psi$ 
and $\varphi, \theta \in \Theta$ such that (3.1) and (3.2) are satisfied and for all $x, y, u, v \in X$ with $g x \geq g u$ and $g y \preceq g \nu$,

$$
\begin{aligned}
& \psi(d(F(x, y), F(u, v))+d(F(y, x), F(v, u))) \\
& \quad \leq \varphi(d(g x, g u)+d(g y, g v))-\theta(d(g x, g u)+d(g y, g v)) .
\end{aligned}
$$

Also, suppose that

(a) $F$ is continuous, or

(b) $X$ is regular.

If there exist $x_{0}, y_{0} \in X$ such that $g x_{0} \preceq F\left(x_{0}, y_{0}\right)$ and $g y_{0} \succeq F\left(y_{0}, x_{0}\right)$, then there exist $x, y \in X$ such that $g x=F(x, y)$ and $g y=F(y, x)$; that is, $g$ and $F$ have a coupled coincidence point in $X$.

Proof We consider the product space $(X \times X, \preceq)$, the metric $d_{1}$ on $X \times X$ and the functions $G: X \times X \longrightarrow X \times X$ and $T: X \times X \longrightarrow X \times X$ as mentioned above. Denote $S=X \times X$. Then $\left(S, d_{1}\right)$ is a complete metric space. By the definition of $G$ and $T$, we have that

(i) $G$ is continuous and nondecreasing; and $T$ is continuous,

(ii) $T(S) \subseteq G(S)$,

(iii) $T$ is $G$-nondecreasing with respect to $\preceq$,

(iv) the pair $(G, T)$ is compatible.

Let $p=(x, y), q=(u, v) \in S=X \times X$ such that $g x \succeq g u$ and $g y \preceq g v$, that is, $(g x, g y) \succeq(g u, g v)$, that is, $G p \succeq G q$. Then (4.1) reduces to

$$
\psi\left(d_{1}(T p, T q)\right) \leq \varphi\left(d_{1}(G p, G q)\right)-\theta\left(d_{1}(G p, G q)\right) .
$$

Now, the existence of $x_{0}, y_{0} \in X$ such that $g x_{0} \preceq F\left(x_{0}, y_{0}\right)$ and $g y_{0} \succeq F\left(y_{0}, x_{0}\right)$ implies the existence of a point $p_{0}=\left(x_{0}, y_{0}\right) \in S$ such that $\left(g x_{0}, g y_{0}\right) \preceq\left(F\left(x_{0}, y_{0}\right), F\left(y_{0}, x_{0}\right)\right)$, that is, $G p_{0} \preceq T p_{0}$. Therefore, the theorem reduces to Theorem 3.1, and hence there exists $w=(x, y) \in S=X \times X$ such that $G w=T w$, that is, $G(x, y)=T(x, y)$, that is, $(g x, g y)=$ $(F(x, y), F(y, x))$, that is, $g x=F(x, y)$ and $g y=F(y, x)$, that is, $(x, y)$ is a coupled coincidence point of $g$ and $F$.

The following corollary is a consequence of Corollary 3.1.

Corollary 4.1 Let $(X, \preceq)$ be a partially ordered set and suppose that there is a metric $d$ on $X$ such that $(X, d)$ is a complete metric space. Let $F: X \times X \rightarrow X$ and $g: X \rightarrow X$ be two mappings such that $g$ is continuous and nondecreasing, $F(X \times X) \subseteq g(X), F$ has the mixed $g$-monotone property on $X$ and the pair $(g, F)$ is commutative. Suppose that there exist $\psi \in \Psi, \varphi, \theta \in \Theta$ such that (3.1), (3.2) and (4.1) are satisfied. Also, suppose that

(a) $F$ is continuous, or

(b) $X$ is regular.

If there exist $x_{0}, y_{0} \in X$ such that $g x_{0} \preceq F\left(x_{0}, y_{0}\right)$ and $g y_{0} \succeq F\left(y_{0}, x_{0}\right)$, then there exist $x, y \in X$ such that $g x=F(x, y)$ and $g y=F(y, x)$; that is, $g$ and $F$ have a coupled coincidence point in $X$.

The following corollary is a consequence of Corollary 3.2. 
Corollary 4.2 Let $(X, \preceq)$ be a partially ordered set and suppose that there is a metric $d$ on $X$ such that $(X, d)$ is a complete metric space. Let $F: X \times X \rightarrow X$ and $g: X \rightarrow X$ be two mappings such that $g$ is continuous and nondecreasing, $F(X \times X) \subseteq g(X), F$ has the mixed $g$-monotone property on $X$ and the pair $(g, F)$ is compatible. Suppose that there exists $\varphi \in \Theta$ such that for any sequence $\left\{x_{n}\right\}$ in $[0, \infty)$ with $x_{n} \rightarrow t>0$,

$$
\varlimsup \varphi\left(x_{n}\right)<t
$$

and for all $x, y, u, v \in X$ with $g x \succeq g u$ and $g y \preceq g v$,

$$
d(F(x, y), F(u, v))+d(F(y, x), F(v, u)) \leq \varphi(d(g x, g u)+d(g y, g v)) .
$$

Also, suppose that

(a) $F$ is continuous, or

(b) $X$ is regular.

If there exist $x_{0}, y_{0} \in X$ such that $g x_{0} \preceq F\left(x_{0}, y_{0}\right)$ and $g y_{0} \succeq F\left(y_{0}, x_{0}\right)$, then there exist $x, y \in X$ such that $g x=F(x, y)$ and $g y=F(y, x)$; that is, $g$ and $F$ have a coupled coincidence point in $X$.

The following corollary is a consequence of Corollary 3.3.

Corollary 4.3 Let $(X, \preceq)$ be a partially ordered set and suppose that there is a metric $d$ on $X$ such that $(X, d)$ is a complete metric space. Let $F: X \times X \longrightarrow X$ and $g: X \rightarrow X$ be two mappings such that $g$ is continuous and nondecreasing, $F(X \times X) \subseteq g(X)$, F has the mixed $g$-monotone property on $X$ and the pair $(g, F)$ is compatible. Suppose that there exist $\psi \in \Psi$ and $k \in[0,1)$ such that for all $x, y, u, v \in X$ with $g x \succeq$ gu and $g y \preceq g v$,

$$
\psi(d(F(x, y), F(u, v))+d(F(y, x), F(v, u))) \leq k \psi(d(g x, g u)+d(g y, g v)) .
$$

Also, suppose that

(a) $F$ is continuous, or

(b) $X$ is regular.

If there exist $x_{0}, y_{0} \in X$ such that $g x_{0} \preceq F\left(x_{0}, y_{0}\right)$ and $g y_{0} \succeq F\left(y_{0}, x_{0}\right)$, then there exist $x, y \in X$ such that $g x=F(x, y)$ and $g y=F(y, x)$; that is, $g$ and $F$ have a coupled coincidence point in $X$.

The following corollary is a consequence of Corollary 3.4.

Corollary 4.4 Let $(X, \preceq)$ be a partially ordered set and suppose that there is a metric $d$ on $X$ such that $(X, d)$ is a complete metric space. Let $F: X \times X \longrightarrow X$ and $g: X \longrightarrow X$ be two mappings such that $g$ is continuous and nondecreasing, $F(X \times X) \subseteq g(X)$, F has the mixed $g$-monotone property on $X$ and the pair $(g, F)$ is compatible. Suppose that there exist $\psi \in \Psi$ and $\theta \in \Theta$ such that for any sequence $\left\{x_{n}\right\}$ in $[0, \infty)$ with $x_{n} \longrightarrow t>0, \underline{\lim } \theta\left(x_{n}\right)>0$ and for all $x, y, u, v \in X$ with $g x \succeq$ gu and $g y \preceq g v$,

$$
\begin{aligned}
& \psi(d(F(x, y), F(u, v))+d(F(y, x), F(v, u))) \\
& \quad \leq \psi(d(g x, g u)+d(g y, g v))-\theta(d(g x, g u)+d(g y, g v)) .
\end{aligned}
$$


Also, suppose that

(a) $F$ is continuous, or

(b) $X$ is regular.

If there exist $x_{0}, y_{0} \in X$ such that $g x_{0} \preceq F\left(x_{0}, y_{0}\right)$ and $g y_{0} \succeq F\left(y_{0}, x_{0}\right)$, then there exist $x, y \in X$ such that $g x=F(x, y)$ and $g y=F(y, x)$; that is, $g$ and $F$ have a coupled coincidence point in $X$.

Remark 4.1 The above result is also true if the arguments of $\psi$ and $\theta$ in (4.2) are replaced by their half values, that is, when (4.2) is replaced by

$$
\begin{aligned}
& \psi\left(\frac{d(F(x, y), F(u, v))+d(F(y, x), F(v, u))}{2}\right) \\
& \quad \leq \psi\left(\frac{d(g x, g u)+d(g y, g v)}{2}\right)-\theta\left(\frac{d(g x, g u)+d(g y, g v)}{2}\right) .
\end{aligned}
$$

In this case, we can write $\psi_{1}(t)=\psi\left(\frac{t}{2}\right)$ and $\theta_{1}(t)=\theta\left(\frac{t}{2}\right)$ and proceed with the same proof by replacing $\psi, \theta$ by $\psi_{1}, \theta_{1}$ respectively. Then we obtain a generalization of Theorem 2 of Berinde in [10].

The following corollary is a consequence of Corollary 3.5.

Corollary 4.5 Let $(X, \preceq)$ be a partially ordered set and suppose that there is a metric d on $X$ such that $(X, d)$ is a complete metric space. Let $F: X \times X \longrightarrow X$ and $g: X \rightarrow X$ be two mappings such that $g$ is continuous and nondecreasing, $F(X \times X) \subseteq g(X)$, F has the mixed $g$-monotone property on $X$ and the pair $(g, F)$ is compatible. Suppose that there exists $\theta \in \Theta$ such that for any sequence $\left\{x_{n}\right\}$ in $[0, \infty)$ with $x_{n} \longrightarrow t>0, \underline{\lim } \theta\left(x_{n}\right)>0$ and for all $x, y, u, v \in X$ with $g x \succeq$ gu and $g y \preceq g v$,

$$
d(F(x, y), F(u, v))+d(F(y, x), F(v, u)) \leq d(g x, g u)+d(g y, g v)-\theta(d(g x, g u)+d(g y, g v)) .
$$

Also, suppose that

(a) $F$ is continuous, or

(b) $X$ is regular.

If there exist $x_{0}, y_{0} \in X$ such that $g x_{0} \preceq F\left(x_{0}, y_{0}\right)$ and $g y_{0} \succeq F\left(y_{0}, x_{0}\right)$, then there exist $x, y \in X$ such that $g x=F(x, y)$ and $g y=F(y, x)$; that is, $g$ and $F$ have a coupled coincidence point in $X$.

The following corollary is a consequence of Corollary 3.6.

Corollary 4.6 Let $(X, \preceq)$ be a partially ordered set and suppose that there is a metric $d$ on $X$ such that $(X, d)$ is a complete metric space. Let $F: X \times X \rightarrow X$ and $g: X \rightarrow X$ be two mappings such that $g$ is continuous and nondecreasing, $F(X \times X) \subseteq g(X)$, F has the mixed $g$-monotone property on $X$ and the pair $(g, F)$ is compatible. Assume that there exists $k \in[0,1)$ such that for all $x, y, u, v \in X$ with $g x \succeq g u, g y \preceq g \nu$,

$$
d(F(x, y), F(u, v))+d(F(y, x), F(v, u)) \leq k[d(g x, g u)+d(g y, g v)] .
$$


(a) $F$ is continuous, or

(b) $X$ is regular.

If there exist $x_{0}, y_{0} \in X$ such that $g x_{0} \preceq F\left(x_{0}, y_{0}\right)$ and $g y_{0} \succeq F\left(y_{0}, x_{0}\right)$, then there exist $x, y \in X$ such that $g x=F(x, y)$ and $g y=F(y, x)$; that is, $g$ and $F$ have a coupled coincidence point in $X$.

The following theorem is a consequence of Theorem 3.2.

Theorem 4.2 Let $(X, \preceq)$ be a partially ordered set and suppose that there is a metric $d$ on $X$ such that $(X, d)$ is a complete metric space. Consider the mappings $F: X \times X \rightarrow X$ and $g: X \rightarrow X$ such that $F(X \times X) \subseteq g(X)$, F has the mixed $g$-monotone property on $X$ and $G(X)$ is closed in $X$. Suppose that there exist $\psi \in \Psi$ and $\varphi, \theta \in \Theta$ such that (3.1), (3.2) and (4.1) are satisfied. Also, suppose that $X$ is regular.

If there exist $x_{0}, y_{0} \in X$ such that $g x_{0} \preceq F\left(x_{0}, y_{0}\right)$ and $g y_{0} \succeq F\left(y_{0}, x_{0}\right)$, then there exist $x, y \in X$ such that $g x=F(x, y)$ and $g y=F(y, x)$; that is, $g$ and $F$ have a coupled coincidence point in $X$.

The following theorem is a consequence of Theorem 3.3.

Theorem 4.3 In addition to the hypotheses of Theorems 4.1 and 4.2, in both of the theorems, suppose that for every $(x, y),\left(x^{*}, y^{*}\right) \in X \times X$, there exists $a(u, v) \in X \times X$ such that $(F(u, v), F(v, u))$ is comparable to $(F(x, y), F(y, x))$ and $\left(F\left(x^{*}, y^{*}\right), F\left(y^{*}, x^{*}\right)\right)$, and also the pair $(g, F)$ is weakly compatible. Then $g$ and $F$ have a unique coupled common fixed point; that is, there exists a unique $(x, y) \in X \times X$ such that $x=g x=F(x, y)$ and $y=g y=F(y, x)$.

Example 4.1 Let $X=[0, \infty)$. Then $(X, \leq)$ is a partially ordered set with the natural ordering of real numbers. Let $d(x, y)=|x-y|$ for $x, y \in X$. Then $(X, d)$ is a complete metric space.

Let $g: X \rightarrow X$ be given by $g x=x^{2}$ for all $x \in X$. Also, consider

$$
F: X \times X \rightarrow X, \quad F(x, y)= \begin{cases}\frac{1}{3}\left(x^{2}-y^{2}\right) & \text { if } x, y \in X, x \geq y, \\ 0 & \text { if } x \leq y\end{cases}
$$

which obeys the mixed $g$-monotone property.

Let $\left\{x_{n}\right\}$ and $\left\{y_{n}\right\}$ be two sequences in $X$ such that

$$
\lim _{n \rightarrow \infty} F\left(x_{n}, y_{n}\right)=\lim _{n \rightarrow \infty} g x_{n}=a, \lim _{n \rightarrow \infty} F\left(y_{n}, x_{n}\right)=\lim _{n \rightarrow \infty} g y_{n}=b .
$$

Then, obviously, $a=0$ and $b=0$.

Now, for all $n \geq 0, g x_{n}=x_{n}^{2}, g y_{n}=y_{n}^{2}$, while

$$
F\left(x_{n}, y_{n}\right)=\left\{\begin{array}{ll}
\frac{1}{3}\left(x_{n}^{2}-y_{n}^{2}\right) & \text { if } x_{n} \geq y_{n}, \\
0 & \text { if } x_{n} \leq y_{n},
\end{array} \quad F\left(y_{n}, x_{n}\right)= \begin{cases}\frac{1}{3}\left(y_{n}^{2}-x_{n}^{2}\right) & \text { if } y_{n} \geq x_{n} \\
0 & \text { if } y_{n} \leq x_{n} .\end{cases}\right.
$$

Then it follows that

$$
\lim _{n \rightarrow \infty} d\left(g F\left(x_{n}, y_{n}\right), F\left(g x_{n}, g y_{n}\right)\right)=0, \quad \lim _{n \rightarrow \infty} d\left(g F\left(y_{n}, x_{n}\right), F\left(g y_{n}, g x_{n}\right)\right)=0
$$


Hence, the pair $(g, F)$ is compatible in $X$.

Let $x_{0}=0$ and $y_{0}=c(>0)$ be two points in $X$. Then

$$
g\left(x_{0}\right)=g(0)=0=F(0, c)=F\left(x_{0}, y_{0}\right), \quad g\left(y_{0}\right)=g(c)=c^{2} \geq \frac{c^{2}}{3}=F(c, 0)=F\left(y_{0}, x_{0}\right) .
$$

Let $\psi, \varphi, \theta:[0, \infty) \longrightarrow[0, \infty)$ be given respectively by the formulas

$$
\psi(t)=t^{2}, \quad \varphi(t)=\left\{\begin{array}{ll}
\frac{1}{2}[t]^{2} & \text { if } 3<t<4, \\
\frac{4}{9} t^{2} & \text { otherwise, }
\end{array} \quad \theta(t)= \begin{cases}\frac{1}{9}[t]^{2} & \text { if } 3<t<4, \\
0 & \text { otherwise }\end{cases}\right.
$$

Then $\psi, \varphi$ and $\theta$ have the properties mentioned in Theorem 4.1.

We now verify inequality (4.1) of Theorem 4.1.

We take $x, y, u, v \in X$ such that $g x \geq g u$ and $g y \leq g v$, that is, $x^{2} \geq u^{2}$ and $y^{2} \leq v^{2}$.

Let $M=d(g x, g u)+d(g y, g v)=\left|x^{2}-u^{2}\right|+\left|y^{2}-v^{2}\right|=\left(x^{2}-u^{2}\right)+\left(v^{2}-y^{2}\right)$.

The following are the four possible cases.

Case-1: $x \geq y$ and $u \geq v$. Then

$$
\begin{aligned}
& d(F(x, y), F(u, v))+d(F(y, x), F(v, u)) \\
& \quad=d\left(\frac{x^{2}-y^{2}}{3}, \frac{u^{2}-v^{2}}{3}\right)+d(0,0)=\left|\frac{\left(x^{2}-y^{2}\right)-\left(u^{2}-v^{2}\right)}{3}\right| \\
& \quad=\left|\frac{\left(x^{2}-u^{2}\right)+\left(v^{2}-y^{2}\right)}{3}\right|=\frac{\left(x^{2}-u^{2}\right)+\left(v^{2}-y^{2}\right)}{3}=\frac{1}{3} M .
\end{aligned}
$$

Case-2: $x<y$ and $u<v$. Then

$$
\begin{aligned}
& d(F(x, y), F(u, v))+d(F(y, x), F(v, u)) \\
& \quad=d(0,0)+d\left(\frac{y^{2}-x^{2}}{3}, \frac{v^{2}-u^{2}}{3}\right)=\left|\frac{\left(y^{2}-x^{2}\right)-\left(v^{2}-u^{2}\right)}{3}\right| \\
& \quad=\left|\frac{\left(x^{2}-u^{2}\right)+\left(v^{2}-y^{2}\right)}{3}\right|=\frac{\left(x^{2}-u^{2}\right)+\left(v^{2}-y^{2}\right)}{3}=\frac{1}{3} M .
\end{aligned}
$$

Case-3: $x \geq y$ and $u \leq v$. Then

$$
\begin{aligned}
& d(F(x, y), F(u, v))+d(F(y, x), F(v, u)) \\
& \quad=d\left(\frac{x^{2}-y^{2}}{3}, 0\right)+d\left(0, \frac{v^{2}-u^{2}}{3}\right) \\
& \quad=\frac{x^{2}-y^{2}}{3}+\frac{v^{2}-u^{2}}{3}=\frac{\left(x^{2}-u^{2}\right)+\left(v^{2}-y^{2}\right)}{3}=\frac{1}{3} M .
\end{aligned}
$$

Case-4: The case ' $x<y$ and $u>v^{\prime}$ ' is not possible. Under this condition, $x^{2}<y^{2}$ and $u^{2}>$ $v^{2}$. Then by the condition $y^{2} \leq v^{2}$, we have $x^{2}<y^{2} \leq v^{2}<u^{2}$, which contradicts that $x^{2} \geq u^{2}$. In all the above cases, it can be verified that (4.1) is satisfied. Hence the required conditions of Theorem 4.1 are satisfied, and it is seen that $(0,0)$ is a coupled coincidence point of $g$ and $F$ in $X$. Also, the conditions of Theorem 4.3 are satisfied, and it is seen that $(0,0)$ is the unique coupled common fixed point of $g$ and $F$ in $X$. 
Remark 4.2 In the above example, the pair $(g, F)$ is compatible but not commuting so that Corollary 4.1 is not applicable to this example, and hence Theorem 4.1 properly contains its Corollary 4.1.

Remark 4.3 As discussed in Remarks 3.2-3.5, Theorem 4.1 properly contains its Corollaries 4.2-4.6.

Remark 4.4 Theorem 4.1 properly contains its Corollary 4.6, which is an extension of Theorem 3 of Berinde [9], and Theorems 2.1 and 2.2 of Bhaskar and Lakshmikantham [8]. Therefore, Theorem 4.1 is an actual extension over Theorem 3 of Berinde [9] and Theorems 2.1 and 2.2 of Bhaskar and Lakshmikantham [8].

Example 4.2 Let $X=[0,1]$. Then $(X, \leq)$ is a partially ordered set with the natural ordering of real numbers. Let $d(x, y)=|x-y|$ for $x, y \in X$. Then $(X, d)$ is a metric space with the required properties of Theorem 4.2.

Let $F: X \times X \rightarrow X, F(x, y)=1$ for $(x, y) \in X \times X$. Let $g: X \rightarrow X$ be given by the formula

$$
g x= \begin{cases}1 & \text { if } x \text { is rational } \\ \frac{1}{2} & \text { if } x \text { is irrational. }\end{cases}
$$

Then $F$ and $g$ have the properties mentioned in Theorems 4.2.

Let $\psi, \varphi, \theta:[0, \infty) \longrightarrow[0, \infty)$ be given respectively by the formulas

$$
\psi(t)=t^{2}, \quad \varphi(t)=\left\{\begin{array}{ll}
\frac{1}{2}[t]^{2} & \text { if } 3<t<4, \\
\frac{4}{9} t^{2} & \text { otherwise, }
\end{array} \quad \theta(t)= \begin{cases}\frac{1}{9}[t]^{2} & \text { if } 3<t<4 \\
0 & \text { otherwise }\end{cases}\right.
$$

Then $\psi, \varphi$ and $\theta$ have the properties mentioned in Theorem 4.2.

All the required conditions of Theorem 4.2 are satisfied. It is seen that every $(x, y) \in$ $X \times X$, where both $x$ and $y$ are rational, is a coupled coincidence point of $g$ and $F$ in $X$. Also, the conditions of Theorem 4.3 are satisfied and it is seen that $(1,1)$ is the unique coupled common fixed point of $g$ and $F$ in $X$.

Remark 4.5 In the above example, the function $g$ is not continuous. Therefore, Theorem 4.1 is not applicable to the above example.

Remark 4.6 In some recent papers [30,31] it has been proved that some of the contractive conditions involving continuous control functions are equivalent. Here two of our control functions are discontinuous. Therefore, the contraction we use here is not included in the class of contractions addressed by Aydi et al. [30] and Jachymski [31].

Competing interests

The authors declare that they have no competing interests.

Authors' contributions

The authors completed the paper together. All authors read and approved the final manuscript.

Author details

'Department of Mathematics, Bengal Engineering and Science University, Shibpur, Howrah, West Bengal 711103, India. ${ }^{2}$ Department of Mathematics, Bengal Institute of Technology, Kolkata, West Bengal 700150, India. ${ }^{3}$ Faculty of Applied

Sciences, University Politehnica of Bucharest, 313 Splaiul Independentei, Bucharest, 060042, Romania. 


\section{Acknowledgements}

The work is partially supported by the Council of Scientific and Industrial Research, India (No. 25(0168)/09/EMR-II). Professor BS Choudhury gratefully acknowledges the support.

Received: 12 March 2013 Accepted: 24 May 2013 Published: 11 June 2013

\section{References}

1. Turinici, M: Abstract comparison principles and multivariable Gronwall-Bellman inequalities. J. Math. Anal. Appl. 117, 100-127 (1986)

2. Choudhury, BS, Kundu, $\mathrm{A}:(\psi, \alpha, \beta)$-weak contractions in partially ordered metric spaces. Appl. Math. Lett. 25(1), 6-10 (2012)

3. Harjani, J, Sadarangani, K: Fixed point theorems for weakly contractive mappings in partially ordered sets. Nonlinear Anal. 71, 3403-3410 (2009)

4. Harjani, J, Sadarangani, K: Generalized contractions in partially ordered metric spaces and applications to ordinary differential equations. Nonlinear Anal. 72, 1188-1197 (2010)

5. Nieto, JJ, Lopez, R: Contractive mapping theorems in partially ordered sets and applications to ordinary differential equations. Order 22, 223-239 (2005)

6. Ran, ACM, Reurings, MCB: A fixed point theorem in partially ordered sets and some applications to matrix equations. Proc. Am. Math. Soc. 132, 1435-1443 (2004)

7. Guo, D, Lakshmikantham, V: Coupled fixed points of nonlinear operators with applications. Nonlinear Anal. 11(5), 623-632 (1987)

8. Gnana Bhaskar, T, Lakshmikantham, V: Fixed point theorems in partially ordered metric spaces and applications. Nonlinear Anal. 65, 1379-1393 (2006)

9. Berinde, V: Generalized coupled fixed point theorems for mixed monotone mappings in partially ordered metric spaces. Nonlinear Anal. 74(18), 7347-7355 (2011)

10. Berinde, $\mathrm{V}$ : Coupled fixed point theorems for $\phi$-contractive mixed monotone mappings in partially ordered metric spaces. Nonlinear Anal. 75, 3218-3228 (2011)

11. Berinde, V: Coupled coincidence point theorems for mixed monotone nonlinear operators. Comput. Math. Appl. 64, 1770-1777 (2012)

12. Choudhury, BS, Kundu, A: A coupled coincidence point result in partially ordered metric spaces for compatible mappings. Nonlinear Anal. 73, 2524-2531 (2010)

13. Choudhury, BS, Maity, P: Coupled fixed point results in generalized metric spaces. Math. Comput. Model. 54, 73-79 (2011)

14. Choudhury, BS, Metiya, N, Kundu, A: Coupled coincidence point theorems in ordered metric spaces. Ann. Univ. Ferrara 57, 1-16 (2011)

15. Harjani, J, López, B, Sadarangani, K: Fixed point theorems for mixed monotone operators and applications to integral equations. Nonlinear Anal. 74, 1749-1760 (2011)

16. Karapınar, E, Luong, NV, Thuan, NX: Coupled coincidence points for mixed monotone operators in partially ordered metric spaces. Arab. J. Math. 1, 329-339 (2012)

17. Lakshmikantham, V, Ćirić, Lj: Coupled fixed point theorems for nonlinear contractions in partially ordered metric spaces. Nonlinear Anal. 70, 4341-4349 (2009)

18. Luong, NV, Thuan, NX: Coupled fixed points in partially ordered metric spaces and application. Nonlinear Anal. 74 983-992 (2011)

19. Samet, B: Coupled fixed point theorems for generalized Meir-Keeler contraction in partially ordered metric spaces. Nonlinear Anal. 72, 4508-4517 (2010)

20. Shatanawi, W, Samet, B, Abbas, M: Coupled fixed point theorems for mixed monotone mappings in ordered partial metric spaces. Math. Comput. Model. 55(3-4), 680-687 (2012)

21. Chen, YZ: Existence theorems of coupled fixed points. J. Math. Anal. Appl. 154, 142-150 (1991)

22. Kadelburg, Z, Pavlović, M, Radenović, S: Common fixed point theorems for ordered contractions and quasicontractions in ordered cone metric spaces. Comput. Math. Appl. 59, 3148-3159 (2010)

23. Jungck, G: Compatible mappings and common fixed points. Int. J. Math. Math. Sci. 9, 771-779 (1986)

24. Jungck, G, Rhoades, BE: Fixed point for set-valued functions without continuity. Indian J. Pure Appl. Math. 29(3), 227-238 (1998)

25. Khan, MS, Swaleh, M, Sessa, S: Fixed points theorems by altering distances between the points. Bull. Aust. Math. Soc. 30, 1-9 (1984)

26. Banach, S: Sur les opérations dans les ensembles abstraits et leur application aux équations intégrales. Fundam. Math. 3, 133-181 (1922)

27. Dutta, PN, Choudhury, BS: A generalisation of contraction principle in metric spaces. Fixed Point Theory Appl. 2008 Article ID 406368 (2008)

28. Jungck, G: Commuting maps and fixed points. Am. Math. Mon. 83, 261-263 (1976)

29. Rhoades, BE: Some theorems on weakly contractive maps. Nonlinear Anal. 47(4), 2683-2693 (2001)

30. Aydi, H, Karapınar, E, Samet, B: Remarks on some recent fixed point theorems. Fixed Point Theory Appl. 76, Article ID $2012(2012)$

31. Jachymski, J: Equivalent conditions for generalized contractions on (ordered) metric spaces. Nonlinear Anal. 74 768-774 (2011) 